\title{
Low SNR Capacity of FSO Links over Gamma-Gamma Atmospheric Turbulence Channels
}

\author{
Fatma Benkhelifa, Zouheir Rezki, IEEE Member, and Mohamed-Slim Alouini, IEEE Fellow \\ Computer, Electrical and Mathematical Science and Engineering (CEMSE) Division \\ King Abdullah University of Science and Technology (KAUST) \\ Thuwal, Makkah Province, Saudi Arabia \\ \{fatma.benkhelifa,zouheir.rezki,slim.alouini\}@kaust.edu.sa
}

\begin{abstract}
In this paper, we study the ergodic capacity of free space optical communication systems over Gamma-Gamma atmospheric turbulence fading channels with perfect channel state information at both the transmitter and the receiver. In our framework, we mainly focus on the low signal-to-noise ratio range and show that the ergodic capacity scales proportionally to $S N R \log ^{4}(1 / S N R)$. We show also that one-bit CSI feedback at the transmitter is enough to achieve this capacity using an on-off power control scheme.
\end{abstract}

Index Terms-FSO, Gamma-Gamma distribution, ergodic capacity, water filling, On-Off power control, low SNR.

\section{INTRODUCTION}

Free-space optical (FSO) communication systems provide high data rates over short distances and are cost effective. FSO communications may be preferred to its counterpart radio frequency $(\mathrm{RF})$ communications in the sense that they are more secure and alleviate the undesirable frequency spectrum segmentation. Moreover, FSO communications have been proposed in order to complement RF communications in hybrid (RF-FSO) systems. However, the performance of the optical channels depends on the external atmospheric conditions caused by the variations of the refractive index. Indeed, the performance of the laser beam can be easily diminished by turbulence induced scintillation as it propagates in the atmosphere [1]-[5]. In order to evaluate the impact of atmospheric turbulence, various models have been proposed to describe the optical wireless channel statistical characteristics. While the log-normal distribution has been found to be the most suitable for the weak-to-moderate turbulence channels, the Gamma-Gamma (GG) distribution has been found to be the most suitable modeling of the optical channels for moderateto-strong turbulence channels [1]-[11]. This distribution is produced from the product of two Gamma random variables and is also known as the generalized $\mathrm{K}$ distribution.

The ergodic capacity is an important figure giving an important benchmark on the data rate that can be supported by the communication link. In RF communications, much interest has been conducted in studying the performance limits of any communication link at low power regime (or equivalently low signal-to-noise ratio (SNR)) [12]-[14]. In [15], upper and lower bounds on the capacity are derived where the channel has a nonnegative input corrupted by an additive white Gaussian noise and constrained to both an average and a peak power constraint. The authors show that the ratio between the upper and lower bounds tends to one when the average power tends to zero. They also show that when only an average power constraint is adopted, the ratio tends to $2 \sqrt{2}$ as the average power tends to zero. However, to the best of our knowledge, no explicit characterization of the capacity where the input is constrained to an average power constraint in the low power regime was investigated in FSO communications. Motivated by this, we provide in this letter a thorough analysis of the ergodic capacity of the FSO channel subject to GG atmospheric turbulence and using optimal power and rate adaptation (OPRA) in the low SNR regime.

A closed form expression for the average capacity using optimal rate adaptation (ORA) is derived for the GG atmo- spheric turbulence in [7]-[9]. A closed form expression of the average capacity for the Log-Normal atmospheric turbulence has been also derived in [7], [9]. A closed form expression for the outage probability is derived for the cases when the scintillation follows a log-normal probability distribution over weak atmospheric turbulence channels and GG probability distribution over moderate-to-strong atmospheric turbulence channels in [7], [10]. In [6], Gappmair has derived closedform expression of the average capacity of FSO channels in turbulent atmosphere for adaptation policies other than ORA, such as the OPRA, channel inversion with fixed rate (CIFR) and truncated channel inversion with fixed rate (TIFR). In [11], a new upper bound on the capacity of power and bandwidth constrained optical wireless links over GG atmospheric turbulence channels, when on-off keying (OOK) formats are used. However, most of the proposed closed-form expressions in the literature do not give much insight because of the presence of the Meijer's G-function. Moreover, the capacity for the OPRA policy in [6] depends on the optimized cutoff SNR that itself depends on the average SNR and it is not clear how the capacity depends on the SNR. In order to better understand this dependence, we consider the asymptotic behavior of the capacity and characterize its dependence in the low SNR regime assuming OPRA and when the optimized cut-off SNR (or the Lagrange multiplier in our framework) is obtained using a water-filing policy for power adaptation. We also propose an on-off scheme that achieves the capacity at asymptotically low SNR regime and that only requires one bit feedback from the receiver side.

\section{System and Channel Models}

We consider a point-to-point FSO communication system using intensity modulation/direct detection. The laser beam propagates through a direct path in the turbulence channel corrupted at the receiver by an additive white Gaussian noise (AWGN). The channel model can be given by [2], [7], [9]

$$
y=s x+n=\eta I x+n,
$$

where $y$ is the output of the channel, $x$ is the input of the channel, $s=\eta I$ is the instantaneous channel gain, where $I$ is the normalized irradiance and $\eta$ is the effective photocurrent conversion ratio of the receiver, and $n$ is the AWGN with a zero mean and a variance $N_{0}$ normalized to 1 . We suppose that both the receiver and the transmitter have full knowledge of the channel gain. The source is subject to an average electrical power constraint $\mathbb{E}_{x \mid I}\left[|x|^{2}\right] \leq P_{\text {avg }}$ where $\mathbb{E}[\cdot]$ is denoting the expectation operator, and $P_{\text {avg }}$ may also be seen as the average transmit SNR as we have a normalized noise. Note that, for most practical optical sources, it is the average power constraints that is more restrictive that the peak power constraints [16]. We mainly focus on asymptotically low SNR, i.e. $P_{\text {avg }} \rightarrow 0$.

For moderate-to-strong turbulence, the irradiance is modeled by a GG distribution [4], [11] whose probability density function (PDF) is given by

$$
f_{I}(I)=\frac{2(a b)^{\frac{a+b}{2}}}{\Gamma(a) \Gamma(b)} I^{\frac{a+b}{2}-1} K_{a-b}(2 \sqrt{a b I}),
$$


where $K_{v}($.$) is the modified Bessel function of second kind and$ of order $v, \Gamma($.$) is the Gamma function and a \geq 0$ and $b \geq 0$ are the distribution shaping parameters that can be expressed as

$$
a=\left[\exp \left(\frac{0.49 \delta^{2}}{\left(1+0.18 d^{2}+0.56 \delta^{12 / 5}\right)^{7 / 6}}\right)-1\right]^{-1}
$$

and

$$
b=\left[\exp \left(\frac{0.51 \delta^{2}}{\left(1+0.9 d^{2}+0.62 \delta^{12 / 5}\right)^{5 / 6}}\right)-1\right]^{-1}
$$

where $\delta^{2}=1.23 C_{n}^{2} k^{7 / 6} L^{11 / 6}$ is the Rytov variance and $d=\sqrt{k D^{2} / 4 L}$ with $k=2 \pi / \lambda_{w}$ is the optical wave number, $L$ is the length of the optical link, $D$ is the receiver's aperture diameter and $C_{n}^{2}$ is the altitude-dependent turbulence strength and varying from $10^{-17} \mathrm{~m}^{-2 / 3}$ for weak turbulence to $10^{-13}$ $\mathrm{m}^{-2 / 3}$ for strong turbulence depending on the atmospheric turbulence conditions. The instantaneous power of the equivalent channel gain according to (1) is defined as $\gamma=\frac{(\eta I)^{2}}{N_{0}}=\eta^{2} I^{2}$. This definition is proper to RF fading channels [4]-[9] which emphasizes the performance of the system that depends on the average power of the electrical current, obtained by the conversion from the optical signal [16]. The PDF of $\gamma$ can then be obtained from (2) by a transformation of the random variable I [7], [9]

$$
f_{\gamma}(\gamma)=\frac{(a b)^{\frac{a+b}{2}}}{\Gamma(a) \Gamma(b)} \frac{\gamma^{\frac{a+b}{4}}-1}{\Omega^{\frac{a+b}{4}}} K_{a-b}\left(2 \sqrt{a b \sqrt{\frac{\gamma}{\Omega}}}\right),
$$

where the average power of the equivalent channel gain is equal to $\Omega=(\eta \mathbb{E}[I])^{2}=\eta^{2}$ [9]. The instantaneous optimal power $P(\gamma)$ is given by the water-filling policy [17]

$$
P(\gamma)=\left[\frac{1}{\lambda(S N R)}-\frac{1}{\gamma}\right]^{+},
$$

where $\lambda(S N R)$ is the Lagrange multiplier obtained by satisfying the average power constraint with equality:

$$
P_{\text {avg }}=S N R=\underset{x \mid \gamma}{\mathbb{E}}[P(\gamma)] .
$$

At this point, let us define the function $G(x)$ over $(0, \infty)$ as

$$
G(x)=\mathbb{E}_{\gamma}\left[\left[\frac{1}{x}-\frac{1}{\gamma}\right]^{+}\right],
$$

where $G($.$) is continuous, positive definite and strictly$ monotonically decreasing [14, Lemma 1] and it satisfies $G(\lambda(S N R))=P_{\text {avg }}=S N R$. Next, we focus on asymptotically low SNR regime, i.e., $P_{\text {avg }} \rightarrow 0$, in which it is useful to define $f(x) \approx g(x)$ if and only if $\lim _{x \rightarrow 0} \frac{f(x)}{g(x)}=1$.

\section{Low-SNR Capacity With Perfect CSI-T}

\section{A. Capacity Results}

The ergodic capacity is obtained by averaging $\log (1+P(\gamma) \gamma)$ over $\gamma$ and is given by

$$
\begin{aligned}
C(\lambda(S N R)) & =\int_{0}^{\infty} \log \left(1+\left[\frac{1}{\lambda(S N R)}-\frac{1}{x}\right]^{+} x\right) f_{\gamma}(x) \mathrm{d} x \\
& =\int_{\lambda(S N R)}^{\infty} \log \left(\frac{x}{\lambda(S N R)}\right) f_{\gamma}(x) \mathrm{d} x .
\end{aligned}
$$

The low-SNR capacity of the FSO channel is given in Theorem 1.
Theorem 1: For a FSO communication channel described by (1), with perfect CSI at both the transmitter and the receiver, the low-SNR capacity is given by

$$
\begin{aligned}
C(S N R) & \approx \begin{cases}\frac{\Omega m^{4} S N R}{16(a b)^{2}} W_{0}^{4}\left(\frac{2 \sqrt{a b}}{m \Omega^{1 / 4}}(\tau S N R)^{\frac{-1}{m}}\right), & \text { if } m>0, \\
\frac{\Omega S N R}{16(a b)^{2}} \log ^{4}(\tau S N R), & \text { if } m=\emptyset, 10) \\
\frac{\Omega m^{4} S N R}{16(a b)^{2}} W_{-1}^{4}\left(\frac{2 \sqrt{a b}}{m \Omega^{1 / 4}}(\tau S N R)^{\frac{-1}{m}}\right), & \text { if } m<0 .\end{cases} \\
& \approx \frac{\Omega}{16(a b)^{2}} S N R \log ^{4}\left(\frac{1}{S N R}\right),
\end{aligned}
$$

where $m=\frac{13}{2}-(a+b), \tau=\frac{\Gamma(a) \Gamma(b) \Omega^{\frac{a+b}{4}-\frac{5}{8}}}{2 \sqrt{\pi}(a b)^{\frac{a+b}{2}-\frac{5}{4}}}$, and $W_{0}($.$) and$ $W_{-1}($.$) are the principal branch and the lower branch of the$ Lambert W-function, respectively.

Proof: First, recall $G($.$) is a strictly monotonically in-$ creasing function [14, Lemma 1], i.e. as $S N R \rightarrow 0, \lambda \rightarrow \infty$. Thus, we can use the series expansion of the modified Bessel function of second kind at infinity [18]

$$
K_{v}(x) \approx \sqrt{\frac{\pi}{2 x}} e^{-x}\left(\sum_{k=0}^{n-1}\left(\frac{1}{2 x}\right)^{k} \frac{\Gamma\left(v+k-\frac{1}{2}\right)}{\Gamma\left(v-k-\frac{1}{2}\right) k !}+o\left(\frac{1}{x^{n-1}}\right)\right)(.12)
$$

Then, we can substitute (12) in (9) to obtain

$$
\begin{aligned}
C(\lambda(S N R)) & \approx \frac{\sqrt{\pi}}{2 \Gamma(a) \Gamma(b)} \frac{(a b)^{\frac{a+b}{2}-\frac{1}{4}}}{\Omega^{\frac{a+b}{4}-\frac{1}{8}}} \int_{\lambda}^{\infty} \log \left(\frac{x}{\lambda}\right) x^{\frac{a+b}{4}-\frac{9}{8}} e^{-2 \sqrt{a b \sqrt{\frac{x}{\Omega}}}} \mathrm{d} x, \\
& \approx \frac{\sqrt{\pi}}{2 \Gamma(a) \Gamma(b)} \frac{(a b)^{\frac{a+b}{2}-\frac{1}{4}}}{\Omega^{\frac{a+b}{4}-\frac{1}{8}}} I_{C}(\lambda),
\end{aligned}
$$

with

$I_{C}(\lambda) \approx \frac{1}{\lambda^{\frac{1}{8}}} 2^{4-a-b}(a b)^{\frac{-1}{2}(a+b)} \Omega^{\frac{a+b}{4}} G_{2,3}^{3,0}\left(2 \sqrt{a b \sqrt{\frac{\lambda}{\Omega}}} \mid \begin{array}{c}\frac{3}{2}, \frac{3}{2}, \frac{1}{2}, a+b\end{array}\right)$,

with $G_{p, q}^{m, n}(.$. .) describes the Meijer's G-function [18]. Using series expansion of the Meijer's G-function at infinity [18], we obtain

$$
C(\lambda(S N R)) \approx \frac{1}{\tau} \lambda^{\frac{a+b}{4}-\frac{5}{8}} e^{-2 \sqrt{a b \sqrt{\frac{\lambda}{\Omega}}},}
$$

with $\tau=\frac{\Gamma(a) \Gamma(b) \Omega^{\frac{a+b}{4}-\frac{5}{8}}}{2 \sqrt{\pi}(a b)^{\frac{a+b}{2}-\frac{5}{4}}}$. On the other hand, using (12) in (8) for $x=\lambda(S N R)$, we obtain

$$
\begin{aligned}
S N R & \approx \frac{\sqrt{\pi}}{2 \Gamma(a) \Gamma(b)} \frac{(a b)^{\frac{a+b}{2}-\frac{1}{4}}}{\Omega^{\frac{a+b}{4}-\frac{1}{8}}} \int_{\lambda}^{\infty}\left(\frac{1}{\lambda}-\frac{1}{x}\right) x^{\frac{a+b}{4}-\frac{9}{8}} e^{-2 \sqrt{a b \sqrt{\frac{x}{\Omega}}}} \mathrm{d} x, \\
& \approx \frac{\sqrt{\pi}}{2 \Gamma(a) \Gamma(b)} \frac{(a b)^{\frac{a+b}{2}-\frac{1}{4}}}{\Omega^{\frac{a+b}{4}-\frac{1}{8}}}\left[\frac{1}{\lambda} I_{1}(\lambda)-I_{2}(\lambda)\right],
\end{aligned}
$$

where

$$
\left\{\begin{array}{l}
I_{1}(\lambda)=\frac{4 \Omega^{\frac{a+b}{4}-\frac{1}{8}}}{(2 \sqrt{a b})^{a+b-\frac{1}{2}}} \Gamma\left(a+b-\frac{1}{2} ; 2 \sqrt{a b \sqrt{\frac{\lambda}{\Omega}}}\right), \\
I_{2}(\lambda)=\frac{4 \Omega^{\frac{a+b}{4}-\frac{9}{8}}}{(2 \sqrt{a b})^{a+b-\frac{9}{2}}} \Gamma\left(a+b-\frac{9}{2} ; 2 \sqrt{a b \sqrt{\frac{\lambda}{\Omega}}}\right),
\end{array}\right.
$$

where $\Gamma(.,$.$) is the incomplete Gamma function. Then using$ the series expansion of $\Gamma(.,$.$) function at infinity [18] in (15)$ yields

$$
S N R \approx \frac{1}{\tau} \lambda^{\frac{a+b}{4}-\frac{13}{8}} e^{-2 \sqrt{a b \sqrt{\frac{\lambda}{\Omega}}}} .
$$

From (14) and (17), we can verify that $C(\lambda(S N R))=$ $S N R \lambda(S N R)$. In order to characterize how the capacity scales as function of the SNR, let us first solve (17) to characterize how the Lagrangian multiplier $\lambda(S N R)$ varies in function of 
the SNR. Note that (17) can be put in the form $y=x e^{x}$ and its solution can be expressed as a function of the principal and the lower branches of the Lambert $\mathrm{W}$-function depending on the value of $m=13 / 2-(a+b)$.

- If $m<0$, a solution of (17) can be expressed as function of the lower branch of the Lambert $\mathrm{W}$-function given that $\frac{2 \sqrt{a b}}{m}\left(\frac{\lambda}{\Omega}\right)^{1 / 4}<0$ and it is expressed as

$$
\lambda(S N R) \approx\left[\frac{m \Omega^{\frac{1}{4}}}{2 \sqrt{a b}} W_{-1}\left(\frac{2 \sqrt{a b}}{m \Omega^{\frac{1}{4}}}(\tau S N R)^{\frac{-1}{m}}\right)\right]^{4} .
$$

Although the Lambert $\mathrm{W}$-function is a built-in function in standard software, we express the capacity in terms of the popular $\log ($.$) function using an infinite ladder$ self-mapping technique given in [19]. The solution to the equation $y=x e^{x}$ with $x>1$ or $y>e$ can be expressed as $x=L_{>}(y)$, with $L_{>}(y)=-\log \left(-\frac{-\log \left(\frac{-\log (\ldots)}{y}\right)}{y}\right)$. For instance, the first infinite functional series is $\log (y)$ and hence we have

$$
\lambda(S N R) \approx \frac{m^{4} \Omega}{16(a b)^{2}} \log ^{4}\left(\frac{2 \sqrt{a b}}{m \Omega^{\frac{1}{4}}}(\tau S N R)^{\frac{-1}{m}}\right),
$$

We know that $\lim _{y \rightarrow 0^{-}} \frac{\log (-\beta y)}{\log (-y)}=1$, for any $\beta>0$ and $y<0$ and hence the result in Theorem 1.

- If $m>0$, a solution of (17) can be expressed in function of the principal branch of the Lambert W-function given that $\frac{2 \sqrt{a b}}{m}\left(\frac{\lambda}{\Omega}\right)^{1 / 4}>0$ and it is expressed as

$$
\lambda(S N R) \approx\left[\frac{m \Omega^{\frac{1}{4}}}{2 \sqrt{a b}} W_{0}\left(\frac{2 \sqrt{a b}}{m \Omega^{\frac{1}{4}}}(\tau S N R)^{\frac{-1}{m}}\right)\right]^{4} .
$$

Similar to the previous case, we also express in this case the capacity in terms of the popular $\log ($.$) function. In this$ case, the solution to the equation $y=x e^{x}$ with $x<-1$ or $-1 / e<y<0$ can be expressed as $x=L_{<}(y)$, with $L_{<}(y)=-\log \left(\frac{\log \left(\frac{\log (\ldots)}{-y}\right)}{-y}\right)$. For instance, the first infinite functional series is $\log (-y)$ and hence we have

$$
\lambda(S N R) \approx \frac{m^{4} \Omega}{16(a b)^{2}} \log ^{4}\left(\frac{2 \sqrt{a b}}{m \Omega^{\frac{1}{4}}}(\tau S N R)^{\frac{-1}{m}}\right)
$$

We know that $\lim _{x \rightarrow \infty} \frac{\log (\beta x)}{\log (x)}=1$, for any $\beta>0$ and $x>0$ and hence the result in Theorem 1.

- If $m=0,(17)$ simplifies to

$$
\tau S N R \approx e^{-2 \sqrt{a b}\left(\frac{\lambda}{\Omega}\right)^{1 / 4}},
$$

whose solution is given by

$$
\lambda(S N R) \approx \frac{1}{16(a b)^{2}} \Omega \log ^{4}\left(\frac{1}{\tau S N R}\right),
$$

where $\tau$ is equal to $\frac{\Gamma(a) \Gamma(b) \Omega}{2 \sqrt{\pi}(a b)^{2}}$ when $m=0$.

This completes the proof of Theorem 1 .

The asymptotic characterization of the capacity provided in Theorem 1 states that at low SNR, the energy efficiency defined as the ratio of the transmitted energy in Joules per information nats by the noise variance of an FSO communication system is proportional to the inverse of $\log ^{4}(1 / S N R)$, which means that communicating one nat of information over an FSO link undergoing a GG fading channel requires much lower energy compared to an RF link undergoing Nakagami fading where the energy efficiency with perfect CSI-T scales essentially as $1 / \log (1 / S N R)[14]$.

\section{B. On-Off Power Control Is Asymptotically Optimal}

In this subsection, we will design a practical scheme in order to asymptotically achieve the capacity using the results gained in Theorem 1. Let us consider an on-off power control that transmits whenever $\gamma \geq \lambda(S N R)$ and remains silent otherwise. Thus, given the power constraint, the instantaneous power $P(\gamma)$ is given by

$$
P(\gamma)= \begin{cases}\frac{S N R}{\operatorname{Prob}(\gamma \geq \lambda(S N R))}, & \text { if } \gamma \geq \lambda(S N R) \\ 0, & \text { otherwise, }\end{cases}
$$

The achievable rate in this case is given by

$$
\begin{aligned}
R(S N R) & =\mathbb{E}_{\gamma}[\log (1+P(\gamma) \gamma)] \\
& \geq \int_{\lambda(S N R)}^{\infty} \log \left(1+\lambda(S N R) \frac{S N R}{\operatorname{Prob}(\gamma \geq \lambda(S N R))}\right) f_{\gamma}(t) \mathrm{d} t \\
& =\log \left(1+\frac{\lambda(S N R) S N R}{\operatorname{Prob}(\gamma \geq \lambda(S N R))}\right) \operatorname{Prob}(\gamma \geq \lambda(S N R))(26)
\end{aligned}
$$

where $\operatorname{Prob}(\gamma \geq \lambda(S N R))$ can be written as

$$
\begin{aligned}
\operatorname{Prob}(\gamma \geq \lambda(S N R)) & =\frac{\sqrt{\pi}}{2 \Gamma(a) \Gamma(b)} \frac{(a b)^{\frac{a+b}{2}-\frac{1}{4}}}{\Omega^{\frac{a+b}{4}-\frac{1}{8}}} I_{1}(\lambda), \\
& \approx \frac{1}{2 \tau} \frac{(a b)^{\frac{3}{4}}}{\Omega^{\frac{1}{4}}} \lambda^{\frac{a+b}{4}-\frac{3}{8}} e^{-2 \sqrt{a b \sqrt{\frac{\lambda}{\Omega}}}} .
\end{aligned}
$$

Obviously, (28) is obtained from (27) using (16) and the series expansion of $\Gamma(.,$.$) function at infinity [18]. Then, we can write$

$$
\frac{\lambda(S N R) S N R}{\operatorname{Prob}(\gamma \geq \lambda(S N R))} \approx \frac{2 \Omega^{\frac{1}{4}}}{(a b)^{\frac{3}{4}}} \lambda^{-\frac{1}{4}},
$$

which converges to zero when $\lambda$ goes to infinity. Therefore, given that (29) is a monotonic function of SNR, we can use the asymptotic approximation $\log (x+1) \approx x$ in (26) and deduce that the achievable rate of the on-off scheme is equal to $\lambda(S N R) S N R$. Hence, 1-bit feedback of CSI-T in each fading realization is enough to achieve the asymptotic capacity. This bit contains the result of the outcome of the comparison between $\gamma$ and $\lambda(S N R)$.

\section{Numerical Results}

In this section, we present some selected numerical results illustrating the accuracy of our characterization given in Theorem 1. In Figs. 1 and 2, we consider two different values of the altitude-dependent turbulence strength $C_{n}^{2}$, namely $910^{-15} \mathrm{~m}^{-2 / 3}$ for moderate turbulence and $310^{-14} \mathrm{~m}^{-2 / 3}$ for strong turbulence, respectively. In our analysis, the value of the wavelength has been set to the typically used value, namely $850 \mathrm{~nm}$ [5]. The receiver's aperture diameter and the length of the optical link have been chosen equal to $0.01 \mathrm{~m}$ and $1000 \mathrm{~m}$, respectively. The values of $a$ and $b$ are then computed using Eqs. (3) and (4). The exact capacity is computed using a numerical root finding algorithm to find the Lagrange multiplier $\lambda$ that satisfies the average power constraint in (7), and then the exact capacity is computed using (9). The no CSI case corresponds to the case where we have no knowledge of the irradiance at the transmitter. The asymptotic capacity with Lambert W-function and with Log function have been obtained using (10) and (11) in Theorem 1 with full CSI at both the transmitter and the receiver. In order to better understand our characterization with the Lambert Wfunction in (10), we have also used an infinite ladder selfmapping technique [19]. In Fig. 1, the values of $a$ and $b$ correspond to the case where $a+b-13 / 2>0$. Note from 
this figure that an accurate characterization of the capacity can be obtained using the good approximation of the Lambert Wfunction in (10) with 6 iterations of the infinite ladder function $x_{n}(y)=L_{<}(y)$ for $-1 / e<y<0$, given that $x_{1}(y)=\log (-y)$ and $x_{n}(y)=x_{1}(y)-\log \left(-x_{n-1}(y)\right), n \geq 2$. We mention also that although the log characterization is not very accurate in Fig. 1 in the displayed SNR range, we have verified that as SNR goes to zero, the gap to the exact curve diminishes considerably (not shown here). In Fig. 2, the values of $a$ and $b$ correspond to the case where $a+b-13 / 2<0$. Note from this figure that an accurate characterization of the capacity can again be obtained using the good approximation of the Lambert Wfunction in (10) using only one iteration of the infinite Ladder function $x_{n}(y)=L_{>}(y)$ for $e<y$, given that $x_{1}(y)=\log (y)$ and $x_{n}(y)=x_{1}(y)-\log \left(x_{n-1}(y)\right), n \geq 2$. In the selected numerical results, we can also characterize the gain of the full CSI-T case over the no CSI-T case which is proportional to $\log ^{4}(1 / S N R)$ compared to $\log (1 / S N R)$ in RF communications. This gain might be of appealing practical interest for designer of hybrid $\mathrm{RF} / \mathrm{FSO}$ systems. Furthermore, the on-off achievable rate is also depicted in Figs. 1 and 2, where it can be seen that it is in fact the closest curve to the capacity suggesting that this suboptimal scheme is worth implementing in the low-SNR regime.

\section{Conclusion}

In this paper, we have analyzed the capacity of a FSO wireless communication channel over GG atmospheric turbulence at low SNR and with full CSI at both the transmitter and the receiver. We showed that the capacity scales asymptotically as $\frac{\Omega}{16(a b)^{2}} S N R \log ^{4}(1 / S N R)$. Moreover, an on-off power control scheme has been shown to be asymptotically achieving the capacity using only one-bit CSI feedback at the transmitter. Our framework has not only confirmed the crucial value of CSI-T on the capacity of FSO communications, but has in fact gone beyond to actually state that the gain due to CSI-T essentially equal to $\log (1 / S N R)$ in RF communication, is in fact more substantial in FSO communications.

\section{AcKnowledgements}

Fatma Benkhelifa would like to thank Dr. Arsenia Chorti and Prof. H. Vincent Poor for some valuable discussions and exchanges during her visit to Princeton University in June/July 2012.

\section{REFERENCES}

[1] X. Zhu and J. Kahn, "Free-space optical communication through atmospheric turbulence channels," IEEE Transactions on Communications, vol. 50, no. 8, pp. 1293 - 1300, Aug 2002.

[2] M. A. Al-Habash, L. C. Andrews, and R. L. Phillips, "Mathematica model for the irradiance probability density function of a laser beam propagating through turbulent media," Optical Engineering, vol. 40, pp. 1554-1562, Aug 2001.

[3] L. C. Andrews, M. A. Al-Habash, C. Y. Hopen, and R. L. Phillips, "Theory of optical scintillation: Gaussian-beam wave model," Waves in Random Media, vol. 11, no. 3, pp. 271-291, 2001.

[4] S. Denic, I. Djordjevic, J. Anguita, B. Vasic, and M. Neifeld, "Information theoretic limits for free-space optical channels with and without memory," IEEE/OSA Journal of Lightwave Technology, vol. 26, no. 19, pp. $3376-3384$, Oct. 2008

[5] A. K. Majumdar, "Free-space laser communication performance in the atmospheric channel," Journal of Optical and Fiber Communications Research, vol. 2, pp. 345-396, 2005.

[6] W. Gappmair, "Further results on the capacity of free-space optical channels in turbulent atmosphere," IET Communications, vol. 5, no. 9, pp. $1262-1267$, June 2011.

[7] H. Nistazakis, T. Tsiftsis, and G. Tombras, "Performance analysis of free-space optical communication systems over atmospheric turbulence channels," IET Communications, vol. 3, no. 8, pp. 1402 -1409, August 2009.

[8] H. Nistazakis, G. Tombras, A. Tsigopoulos, E. Karagianni, and M. Fafalios, "Average capacity of wireless optical communication systems over Gamma Gamma atmospheric turbulence channels," in Proc. IEEE International Microwave Symposium Digest (MTT-S'2008), Atlanta, GA, USA, June 2008.

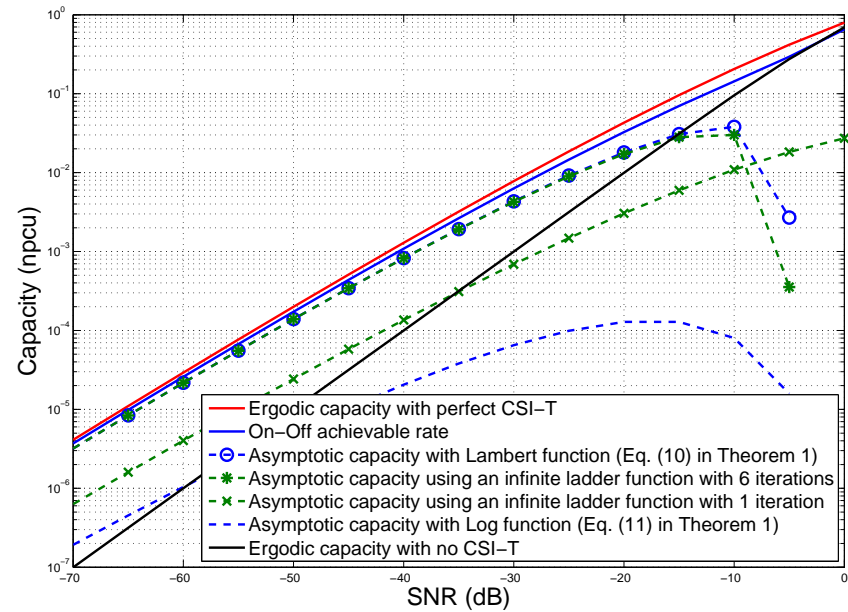

Figure 1. Low-SNR capacity in nats per channel use (npcu) versus SNR for moderate turbulence $C_{n}^{2}=910^{-15} m^{-2 / 3}(a=6.4900$ and $b=7.2092)$.

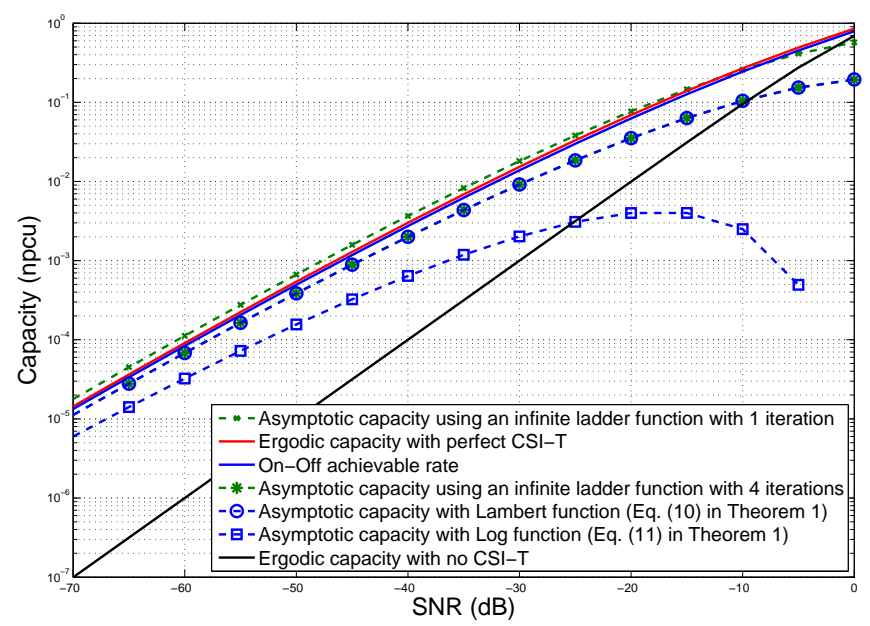

Figure 2. Low-SNR capacity in nats per channel use (npcu) versus SNR for strong turbulence $C_{n}^{2}=310^{-14} \mathrm{~m}^{-2 / 3}(a=2.7454$ and $b=3.0543)$.

[9] H. Nistazakis, E. Karagianni, A. Tsigopoulos, M. Fafalios, and G. Tombras, "Average capacity of optical wireless communication systems over atmospheric turbulence channels," IEEE/OSA Journal of Lightwave Technology, vol. 27, no. 8, pp. 974 -979, April 2009.

[10] H. Li-Qiang, W. Qi, and S. Katsunori, "Outage probability of free space optical communication over atmosphere turbulence," in Proc. WASE International Conference on Information Engineering (ICIE'2010), Beidai, China, Aug. 2010.

[11] A. García-Zambrana, C. Castillo-Vázquez, and B. Castillo-Vázquez, "On the capacity of FSO links over Gamma-Gamma atmospheric turbulence channels using OOK signaling," EURASIP J. Wirel. Commun. Netw. vol. 2010, pp. 64:1-64:11, Jan 2010.

[12] S. Borade and L. Zheng, "Wideband fading channels with feedback," IEEE Transactions on Information Theory, vol. 56, no. 12, pp. 6058 -6065 , Dec. 2010.

[13] S. Verdu, "Spectral efficiency in the wideband regime," IEEE Trans. Information Theory, vol. 48, no. 6, pp. 1319-1343, Sep. 2006.

[14] Z. Rezki and M.-S. Alouini, "On the capacity of Nakagami-m fading channels with full channel state information at low SNR," IEEE Wireless Communications Letters, no. 3, pp. 253 -256, June 2012.

[15] A. Lapidoth, S. Moser, and M. Wigger, "On the capacity of free-space optical intensity channels," IEEE Transactions on Information Theory, vol. 55 , no. 10 , pp. $4449-4461$, Oct. 2009

[16] S. Hranilovic and F. Kschischang, "Optical intensity-modulated direct detection channels: signal space and lattice codes," IEEE Transactions on Information Theory, vol. 49, no. 6, pp. 1385 - 1399, June 2003.

[17] A. Goldsmith and P. Varaiya, "Capacity of fading channels with channel side information," IEEE Transactions on Information Theory, vol. 43 no. 6, pp. 1986 -1992, Nov. 1997.

[18] I. S. Gradshteyn and I. M. Ryzhik, Table of Integrals, Series, and Products, 7th ed. Elsevier/Academic Press, Amsterdam, 2007.

[19] G. Pickett and Y. Millev, "On the analytic inversion of functions, solution of transcendental equations and infinite self-mappings," Journal of Physics A: Mathematical and General, vol. 35, no. 20, p. 4485, 2002. 